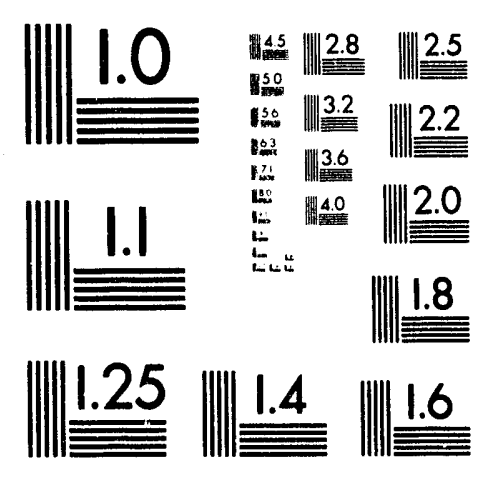



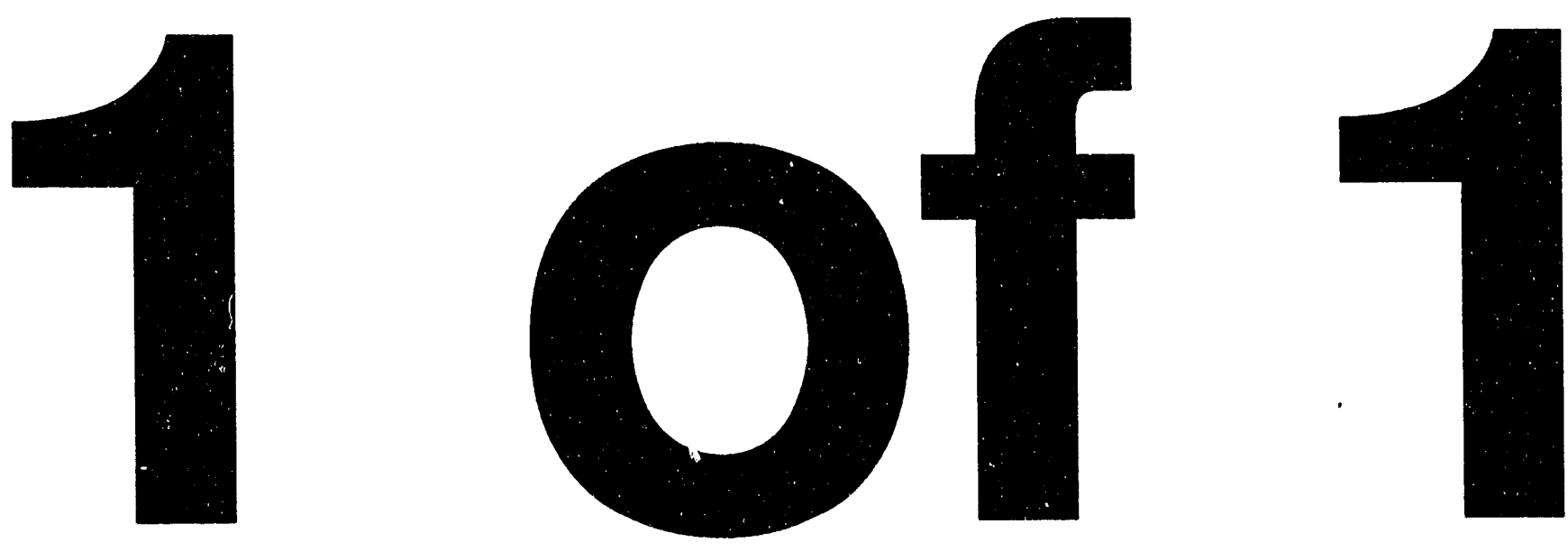
LBL-33292

UC-410

LSGN-123

\title{
PERFORMANCE OF ADVANCED LIGHT SOURCE PARTICLE BEAM DIAGNOSTICS
}

\author{
JM HINKSON \\ ACCELERATOR and FUSION RESEARCH DIVISION \\ Lawrence Berkeley Laboratory \\ University of California \\ Berkeley, CA 94720
}

MAY 1993

This work was supported by the Director, Office of Energy Research, Office of Basic Energy Sciences, Materials Sciences Division, of the U.S. Department of Energy under Contract No. DE-AC03-76SF00098. 


\title{
PERFORMANCE OF ADVANCED LIGHT SOURCE PARTICLE BEAM DIAGNOSTICS*
}

\author{
J. Hinkson \\ Accelerator and Fusion Research Division \\ Lawrence Berkeley Laboratory \\ University of California \\ Berkeley, California 94720
}

\begin{abstract}
The Advanced Light Source (ALS), a third-generation synchrotron radiation facility, is complete. The particle beam diagnostics have been installed and tested. The beam injection systems have been running for two years. We have performance data on beam position monitors, beam intensity monitors, scintillators, beam collimators, a $50 \Omega$ Faraday cup, and broad-band striplines and kickers used in the linac, transport lines, and the booster synchrotron. The single-turn monitoring capability of the booster beam position monitoring system has been particularly useful for studying beam dynamics. Beam diagnostics for the storage ring are being commissioned. In this paper we describe each instrument, show its performance, and outline how the instruments are controlled and their output data displayed.
\end{abstract}

\section{INTRODUCTION}

Recently, the commissioning performance goals of the ALS storage ring were met. Multi-bunch beam intensity has reached 460 $\mathrm{mA}$. Single-bunch current has exceeded the $8 \mathrm{~mA}$ specification by a factor of three. Beam lifetime is dominated by vacuum chamber pressure and is increasing as synchrotron light scrubs the many photon stops. At $100 \mathrm{~mA}$, the lifetime is about 1 hour. The commissioning process went very rapidly. Instrumentation played a critical role.

\section{BEAM INTENSITY MONITORS}

\section{A. Wall Current Monitors}

Two identical monitors are installed, one near the electron gun, and the other in the $1.5 \mathrm{GeV}$ beam transport line connecting the booster and storage rings. The monitor near the electron gun is described. The function of this device is to measure the amplitude and duration of the RF-modulated beam emitted from the electron gun. Beam energy at this location is $120 \mathrm{keV}$. The gun emits from 1 to 20 beam bunches, 2 ns FWHM, spaced at $8 \mathrm{~ns}$, at a $1 \mathrm{~Hz}$ repetition rate. Peak current is betweem 1 and $2 \mathrm{~A}$.

The wall current monitor requires a gap in the beam pipe to intercept the beam image (wall) current. This is provided by a commercially available ceramic insulator fitted to $114 \mathrm{~mm}$ diameter flanges on a $64 \mathrm{~mm}$ diameter beam pipe. The ceramic insulator is approximately $25 \mathrm{~mm}$ long and is coated with a thin layer of chromium on the vacuum side. This coating protects the ceramic from the beam and somewhat damps wave guide modes (important only in the high-energy transport line beam monitor). The

*This work supported by the Director, Office of Energy Research, Office of Basic Energy Sciences, Materials Sciences Division of the U.S. Department of Energy, under Contract No. DE-AC0376SF00098 resistance of the metalization is $60 \Omega$. Twenty, $39 \Omega$, lowinductance, leadless resistors are installed symmetrically around the outside of the ceramic gap. The resistors are soldered to soft copper rings, which are strapped to the metal beam pipe on each side of the gap. The total resistance across the gap is about $1.9 \Omega$.

With a single tap on the resistor belt we noticed undesirable beam position sensitivity during the early part of each bunch. By tapping the resistor belt at four equally spaced locations and summing the voltages in three, 180-degree hybrid combiners, the position sensitivity is considerably reduced. The summed output of the hybrids is displayed on an SCD1000 scan-converter oscilloscope via 60 feet of 0.25 inch Heliax cable. The ALS control system collects oscilloscope trace data over the General Purpose Interface Bus (GPIB) and displays the trace in the control room at $1 \mathrm{~Hz}$. At the oscilloscope input the final sensitivity including cable and hybrid response is about $2.5 \mathrm{~V} / \mathrm{A}$.

High frequency response of the wall current monitor is dominated by the gap resistance and shunt capacitance of $20 \mathrm{pf}$. In our monitor the response is reduced by $3 \mathrm{~dB}$ at $4.2 \mathrm{GHz}$. The beam bunch spectra at the electron gun do not extend beyond about 1 $\mathrm{GHz}$. The upper limit of the hybrid response is $2 \mathrm{GHz}$. The oscilloscope response is down $3 \mathrm{~dB}$ at $1 \mathrm{GHz}$. There is sufficient high frequency response in the system to show relevant beam bunch structure.

Low frequency response of the wall current monitor is dominated by system inductance. The beam pipe is grounded in many locations, creating a low-frequency short circuit across the gap. The limited inductance in these short circuits causes a noticeable baseline tilt in the oscilloscope response when we observe many bunches at $8 \mathrm{~ns}$ intervals. The calculated total inductance across the gap resistance is $130 \mathrm{nH}$. We intend to improve low-frequency response with external frequency compenstation circuits.

\section{B. Faraday Cup}

This device is used to measure total beam charge in the 50 $\mathrm{MeV}$ transport line between the linac and booster. A passive, impedance matched RC network integrates the cup voltage for presentation on a remote digitizing oscilloscope. The wave form is displayed in the control room. We hoped the cup's $50 \Omega$ construction would yield frequency response high enough to resolve adjacent S-band beam bunches. Our aim was to make a straightforward sub-harmonic buncher tuning aid. A rise time of 100 ps or less in a single-shot system was necessary. The best cup rise time we have observed is about $300 \mathrm{ps}$ due to the lumped capacitance of a ceramic gap on the end of the cup. See Figure 2. Although the cup does not have the frequency response we desire, it does accurately quantitize total beam charge. One interesting 
feature of this design is the ceramic break. It supports the coaxial center conductor and permits having an air dielectric. We are not required to use a vacuum coaxial feedthrough. Assembly is quite easy.

4 YINCH FLNGE

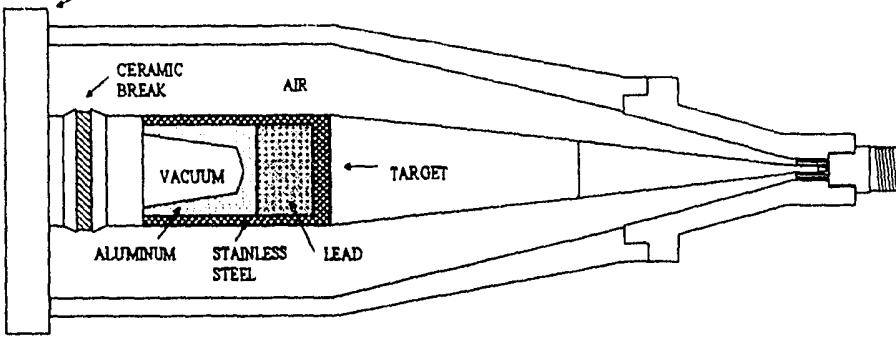

Fig. 2: Drawing of $50 \mathrm{MeV}, 50 \Omega$ Faraday Cup

Recently we tested $11 \mathrm{~mm}$ electrostatic pickup buttons (Ceramex, type ESRF) installed near the cup for their suitability as bunch monitors. The results are encouraging. With the linac bunchers detuned we can observe small adjacent $\$$-band buckets on an SCD 5000 oscilloscope. This instrument is a $5 \mathrm{GHz}$ single-shot digitizer with an effective sampling rate of $200 \mathrm{Gs} / \mathrm{s}$. When the bunchers are properly tuned we observe a large single bunch. System rise time is about 100 ps. Some objectionable ringing is seen following the bunch signal. This may be due to button response and/or wake fields produced in nearby beam pipe discontinuities.

\section{DC Current Transformer (DCCT)}

A Holec DCCT with $100 \mathrm{~mA}$ full-scale sensitivity is used in the booster synchrotron. This device has adequate time response to measure average beam current during the booster acceleration cycle ( $350 \mathrm{~ms}$ ). Unfortunately, the DCCT electronics are quite sensitive to RF. Our DCCT core shield resonates at $40 \mathrm{MHz}$, the 10th harmonic of the booster revolution frequency, and couples RF energy to the core windings. $R F$ filters installed in the cable between the DCCT cores and the electronics have helped reduce the effects of RF pickup. The filters have upset the DCCT sense circuits somewhat. As a result we see interference at $55 \mathrm{~Hz}$ (the core modulation rate) equivalent to $100 \mu \mathrm{A}$ beam current. With a single $1 \mathrm{~mA}$ bunch in the booster we also see RF interference upsetting the DCCT feedback circuits.

A Bergoz parametric current transformer is installed in the storage ring. This instrument has two ranges, $10 \mathrm{~mA}$ and $1 \mathrm{~A}$ full scale $(10 \mathrm{~V}$ ). It also has a di/dt output of $-10 \mathrm{~mA} / \mathrm{s} / \mathrm{V}$ (on the $1 \mathrm{~A}$ range). Noise and offsets sampled at $1 \mathrm{~Hz}$ are equivalent to a few micro amperes of beam, well within ALS requirements. The DCCT shield is a very complex device based on a LEP design [1]. The shield resonates in a $\lambda / 4$ coaxial mode at $40 \mathrm{MHz}$. Beam impedance measurements [2] predict $5.5 \mathrm{~W}$ will be lost in the shield at $50 \mathrm{~mA}$ beam current in a single bunch. So far, DCCT core temperature measurements at all levels of beam current have revealed no temperature rise. We have observed no RF effects on the DCCT performance. The cores and electronics are well shielded. A nearby corrector magnet has sufficient leakage fields to cause objectionable DCCT offsets. We installed a soft iron shield around the DCCT enclosure to eliminate the problem.

In order to corroborate storage ring DCCT readings, a calorimetric experiment was conducted [2] to determine average beam current. The cooling water temperature rise in a photon stop intercepting a known fraction of the total synchrotron light was measured at $300 \mathrm{~mA}$ beam current. The calculated average current from this measurement was $280 \mathrm{~mA}$.

The storage ring DCCT has three output signals, wide-band, low-pass filtered, and di/dt. The wide-band and di/dt signals are fed to a digitizing oscilloscope for control room display. The low-pass filtered and di/dt outputs are fed to 16-bit digitizers in the control system. Computer displays show current plotted against time and calculated beam lifetime.

\section{BEAM POSITION MONITORS}

Beam position monitors (BPMs) [3] are installed in 96 locations in the storage ring, 32 locations in the booster synchrotron, and in 15 places in the linac and transport lines. A detailed description of the BPMs is beyond the scope of this paper. A brief description of storage ring BPMs and their performance to date follows. A block diagram of the BPM electronics is shown in Figure 2.

Each BPM pickup array consists of four button-style electrodes. The electrodes are connected to individual super-heterodyne receivers via Heliax cable. The $500 \mathrm{MHz}$ component of the bunched beam spectrum is selected and mixed down to $50 \mathrm{MHz}$. A broad-band pseudo-synchronous detector converts the signals to base band. The detected signal is fed to three circuits, a video monitor, a fast Analog-to-Digital (A/D) converter, and a $1 \mathrm{kHz}$ low-pass filter. The filtered signal is digitized for calculation of beam position by the difference-over-sum method. These calculations are performed by the on-board Intelligent Local Controller (the basic input-output module for the ALS computer control system). The fast A/D data are fed to first-in-first-out memory where up to 1024 tums of beam data are stored.

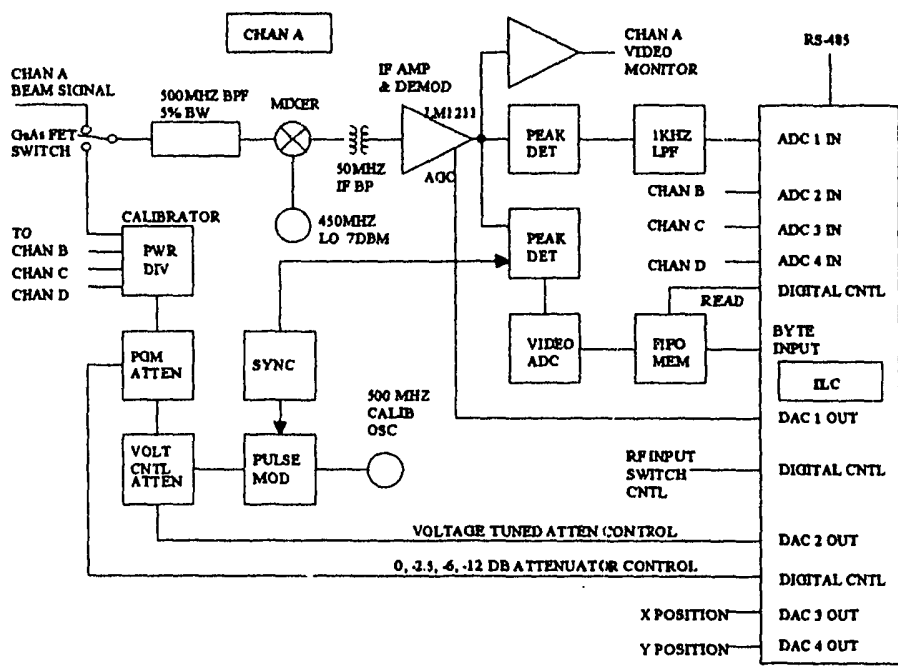

Fig. 2: One of four BPM channels.

When the fast digitizers are stopped, we recover the single-turn data from one or all BPMs. These data are useful for tune measurements, single-shot closed orbit measurements, and other transient beam phenomena. It was these fast $\mathrm{A} / \mathrm{Ds}$ that helped us discover a defective quadrupole magnet in the storage ring lattice. We were having great difficulty getting more than a few turns of beam. Only when single-turn BPM data were compared with the lattice model [4] did we know where to look for the culprit. A quick 
magnet repair was performed, and beam circulated with little tuning.

The BPMs require internal measurement of differential gain and offsets in order to make repeatable measurements to the required $30 \mathrm{~mm}$. For reasons discovered just recently the storage ring BPMs would not perform an internal calibration sequence. Even so, the averaged beam position reacings with stored beam made closed orbit corrections possible to $0.5 \mathrm{~mm}$ rms. With BPM calibration working we expect better than $0.1 \mathrm{~mm}$ performance.

\section{SCINTILLATORS AND TV}

Throughout most of the ALS project we use scintillators made of high-purity alumina doped with chrome. They are referred to as Chromox 6 fluorescent screens and are produced by Morgan Matroc Ltd. in the U.K. The scintillators are UHV compatible and, in over three years of service, we have seen no signs of beam damage. Two zinc oxide scintillators are installed near the electron gun. We found the Chromox 6 scintillators were not usable in this location. Apparently, lack of a conducting substrate caused the scintillators to charge as they stopped the low-energy beam. A distorted image and some flashing resulted.

arly on, with weak beams, we had difficulty obtaining enough inght from Chromox 6 for our CCD TV cameras. This was due to camera optics, number of electron bunches, and rather diffuse beams. TV image frame-grabbing and image enhancement helped. At the suggestion of a visiting colleague [5] we removed the infrared filter from the CCDs and obtained a huge increase in camera response. The scintillators produce strong infrared with a long response tail. The $\mathrm{CCDs}$ are quite sensitive at those long wavelengths.

Our efforts have been to commission the accelerator, and consequently, there has been little attention paid to improving the TV systems. We intend to study scintillator response and determine ways to optimize the scintillator and TV systems for best performance.

\section{BROAD BAND STRIPLINES}

Two broad band stripline pickups [6] are installed in the booster synchrotron. They are referred to as traveling wave electrodes (TWE). They were intended to be part of a fractional tune measurement system. The pickups have good frequency response and adequate coupling to the beam. The kicker however does not have sufficient coupling. We have been unable to excite detectable betatron motion in booster beam with as much as $70 \mathrm{~W}$ driving the kicker. We feel this is due to a rather large tune spread. The booster sextupoles that would reduce the tune spread are not needed for satisfactory beam acceleration. Our plan is to run the sexupoles and attempt more tune measurements. If we are unsuccessful, we will install a more robust kicker.

It is worth noting that booster fractional tune measurements are easily done with the BPM single-turn response and multi-turn data storage. At injection we determine fractional tune by performing an FFT on 1024 sequential turns. At higher energies, when the beam is damped, we excite it with the extraction kicker at low fields and again use the BPM data to determine tune.
We have no striplines, TWEs, or transverse kickers installed in the storage ring at this iime. Consequently, accurate fractional tune measurements he ve been difficult to obtain when we have a stable, damped beam. We have had some limited success tickling the beam horizontally using one of three arrays of LEP BPM buttons, a $100 \mathrm{~W}$ power amplifier, and a tracking generator/spectrum analyzer combination. These buttons are not meant to be kickers. They will be used as pickups for transverse and longitudinal damping systems. We are able to use the single-turn capability of the BPMs to determine tune to 0.01 when the beam is unstable (at injection and at high current). A Tektronix 3052 fast spectrum analyzer displaying BPM spectra or an FFT performed 1024 turns of BPM data show similar results. We plan to install the transverse damping system kickers this year and will use them to excite a damped beam in the future. We expect we will achieve 0.001 tune resolution then.

\section{BEAM COLLIMATORS}

Adjustable two-jaw beam collimators are installed in the lowenergy diagnostics line off the linac and in the line between the linac and booster. A single collimator is used in the diagnostics 'ine and defines a vertical slit. Two collimators in the booster line define a beam aperture. The $50 \mathrm{MeV}$ beam is stopped with 0.5 inch tantalum plates. Stepper motors position the plates with $10 \mu \mathrm{m}$ resolution. Absolute position encoders read position to a few microns

\section{CONCLUSION}

The diagnostics are working well enough to commission and run the accelerators. Some improvements are needed. BPM resident software will be changed to take advantage of the device's ability to calibrate offsets and correct detector nonlinearity. We currently have no way of measuring total beam charge at the exit of the booster. We plan to install an integrating current transformer there. More work will be done on the wide band linac bunch monitor.

\section{REFERENCES}

[1] K. Unser, personal communication to author.

[2] K. Kennedy, personal communication to author

[3] J. Hinkson, "Advanced Light Source Beam Position Monitor," AIP Conference Proceedings No. 252 on Accelerator Instrumentation, Particle and Fields Series $46, \mathrm{pp} .21-41$

[4] J. Bengtsson, M. Meddahi, "Elementary Analysis of BPM Data for the ALS Storage Ring," LBL CBP Tech Note 003, March 1, 1993.

[5] J.C. Denard, personal communication to author.

[6] J. Hinkson, "A Wide Band Slot-Coupled Beam Sensing Electrode for the Advanced Light Source (ALS)," Conference Record of the 1991 IEEE Particle Accelerator Conference, Vol. 2. pp. 1234-1236 

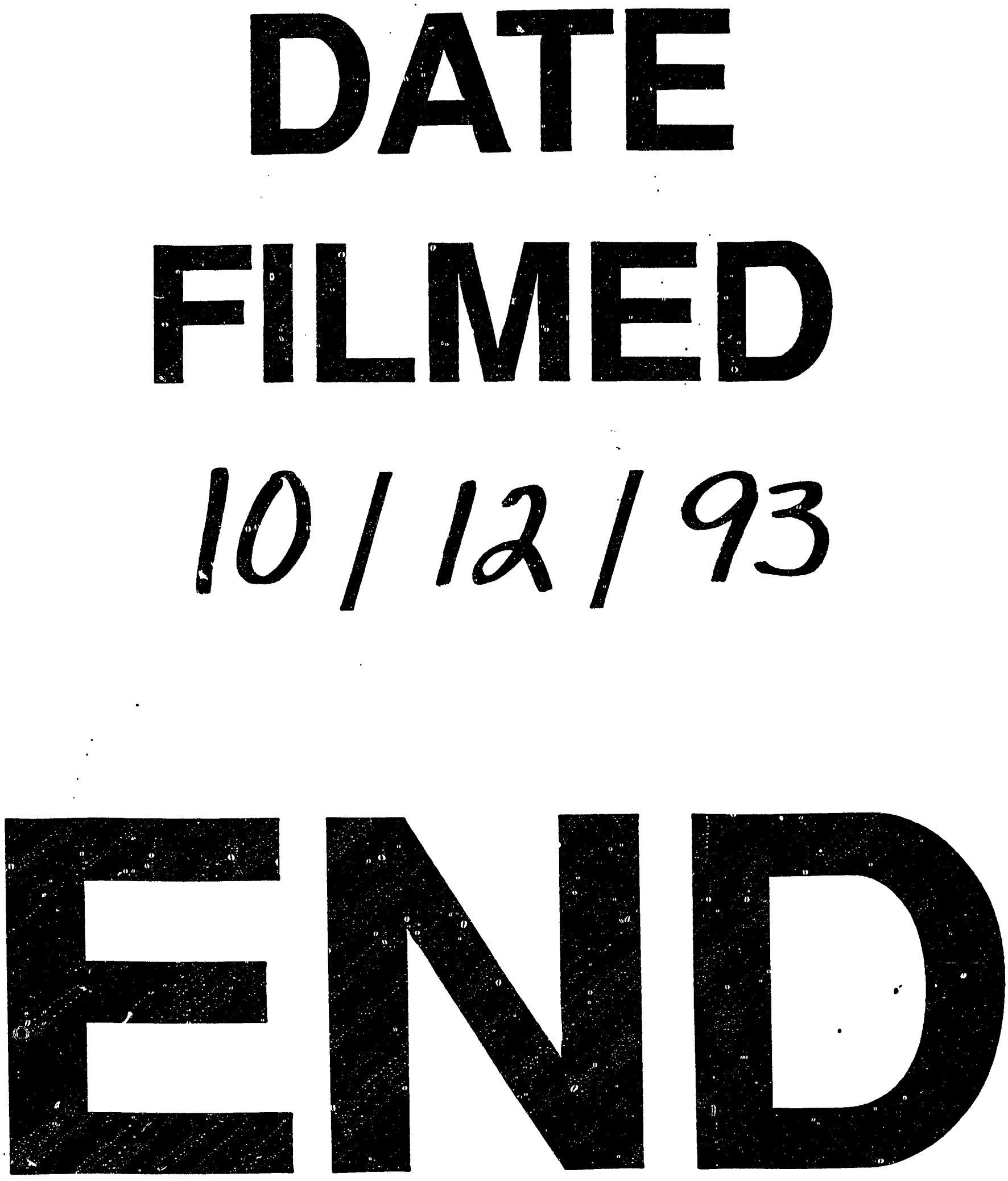
